\section{Surgical Treatment in Traumatic Brain Injury with Critical Glasgow Coma Scale (GCS): Clinical Outcome in a Single Center}

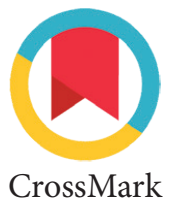

\author{
Tedy Apriawan, ${ }^{1}$ Fitra Fitra ${ }^{2 *}$
}

\title{
ABSTRACT
}

Aims: The goal of this study was to analyze outcomes of the patients underwent surgery for traumatic brain injury (TBI) with critical Glasgow coma scale at Dr. Soetomo General Hospital Surabaya, Indonesia in term of mortality.

Methods: All cases of critical TBI patients with hemorrhage was operated (evacuation craniotomy) from January 2015 - December 2016 at Dr. Soetomo General Hospital Surabaya, Indonesia. The data were retrospectively reviewed from the medical records. Information regarding patient age, sex, diagnoses, Glasgow Coma Scale (GCS), surgery type, and the outcome after surgery was collected and analyzed.

Results: 36 samples were analyzed from the medical records of Dr. Soetomo hospital. The operation was performedfor the patients with epidural hemorrhage (EDH), subdural hemorrhage (SDH) and intracerebral hemorrhage (ICH). In the postoperative period, 83.78\% (30 patients) of patients died and only 16.22\% (6 patients) survived. Survived patients were followed until six months according to Glasgow Outcome Scale (GOS).As regard with the six-month evaluation of GOS, only 2 patients came out with good functional outcome (GOS Extended8), 2 patients came out with lower moderate disability (GOS Extended 5), and 2 patients with dead status (GOS Extended 1).

Conclusions: In this retrospective study, overall $83.78 \%$ (30 patients) patients not survived but $16.22 \%$ (6 patients) survived. Two patients had better functional outcome after six months postoperative. The authors conclude that TBI patients with critical GCS should manage with surgery as indicated since good functional results can be obtained in some cases. Many factors affected the good and bad condition of the patient after craniotomy.

Keywords: Critical GCS, hemorrhage evacuation craniotomy, GOS Extended

Cite This Article: Apriawan T., Fitra, F. 2018. Surgical Treatment in Traumatic Brain Injury with Critical Glasgow Coma Scale (GCS): Clinical Outcome in a Single Center. Indonesian Journal of Neurosurgery 1(1): 28-30. D01:10.15562/ijn.v1i1.3

${ }^{1}$ Neurotrauma Division, Neurosurgery Department, Faculty of Medicine, Universitas Airlangga/ Dr. Soetomo General Hospital Surabaya

${ }^{2}$ Resident of Neurosurgery Department, Faculty of Medicine, Universitas Airlangga/ Dr. Soetomo General Hospital Surabaya
*Correspondence to: Fitra Fitra, Resident of Neurosurgery Department, Faculty of Medicine, Universitas Airlangga/ Dr. Soetomo General Hospital Surabaya afalfitra@gmail.com

\section{INTRODUCTION}

Traumatic brain injury (TBI) is the cause of death and disability among children and adolescents. It was found that the patients who survive often left with persistent impairments that have the potential to impede daily functioning, delay or prevent the attainment of developmental milestones, and subsequently limit future productivity in adulthood. ${ }^{1-4}$ Although hemorrhage evacuation craniotomy has been routinely utilized for management of epidural hemorrhage(EDH), subdural hemorrhage (SDH), and intracerebral hemorrhage (ICH) as indicated, the application of such strategy for traumatic brain injury patients with critical Glasgow Coma Scale (GCS) is limited. ${ }^{1,5-7}$ A study by Chamoun et al. reported favorable outcomes in a study of 189 blunt head trauma patients with GCS score of 3 after aggressively treatment. ${ }^{8}$

In our center, authors performed hemorrhage evacuation craniotomy as indicated in TBI patients with critical GCS. An accurate early prediction of survival and functional outcome appears to be paramount importance and allows for informed counseling of relatives and helps the treating neurosurgeon in deciding the aggressiveness of treatment. In this study, we reported our experience in the treatment of patients with traumatic brain injury presenting with a GCS score of below and equal 5 . The primary objective was to determine the overall mortality outcome of hemorrhage evacuation craniotomy traumatic brain injury patients with critical GCS and to evaluate patients GOS Extended for six months.

\section{MATERIAL AND METHODS}

Authors designed a retrospective cohort study. Chart review had been conducted for all cases of patients with traumatic brain injury presenting with a GCS score of below and equal 5 underwent hemorrhage evacuation craniotomy at our institution from January 2015 to December 2016. Patients with $\mathrm{EDH}, \mathrm{SDH}$, and ICH were considered for study with strict selection criteria further described. We established Preoperative diagnosis based on the clinical presentation and pre-operative CT Scan. As a large neurosurgery center in Indonesia, we have a high volume of patients with TBI, the 
facility, and skilled team to complete hemorrhage evacuation craniotomy. Patients with age below 15 were not deemed suitable candidates for this study. Lesion location, according to hemisphere and lobe involvement, was determined based on CT Scan images obtained and reviewed for each patient with $\mathrm{EDH}, \mathrm{SDH}$ and $\mathrm{ICH}$. Surgery performed within below 6 hours after patients came to the emergency room and being transferred to the operating room. The operation included hemorrhage evacuation craniotomy and decompression which were implemented for several patients. Patient outcomes were determined with GOSE at six months postoperative.

\section{Statistical analysis}

Descriptive statistics were explained as median, mean, and standard deviations for continuous variables and frequency of distribution for categorical variables. Statistical analyses and comparison were not performed.

\section{Medical Treatment}

The medical treatment was performed according to Dr. Soetomo Hospital standard protocol. All patients underwent surgically treatable craniotomy for TBI pathological findings (EDH, SDH, and ICH fulfilled surgical indication). All patients underwent ICP Monitor insertion intraoperative. A decompressive craniectomy in this study was performed due to the decision of operating surgeons based on the intraoperative findings.

\section{RESULTS}

\section{Patient demographics}

Table 1 shows records of 36 patients who underwent hemorrhage evacuation craniotomy between January 2015 to December 2016. Age of the patient approximated by mean age of 34 years old and ranging from 15 to 74 years old by standard deviation of 14.89 years. There was a dominant male (27 male)

\section{Table 1 Characteristics of patients}

\begin{tabular}{lc}
\hline Characteristic & Value* \\
\hline Male & $27(75)$ \\
Ages (years) & \\
$\quad<17$ & $4(11.1)$ \\
$17-25$ & $6(16.6)$ \\
$26-45$ & $19(52.7)$ \\
$>46$ & $7(19.4)$ \\
Pupil Size\& reactivity & \\
Bilateral reactive not dilatated & $24(66.60)$ \\
Bilateral fixed, dilatated & $12(33.3)$ \\
\hline
\end{tabular}

${ }^{*}$ Value are represented as number $(\%)$

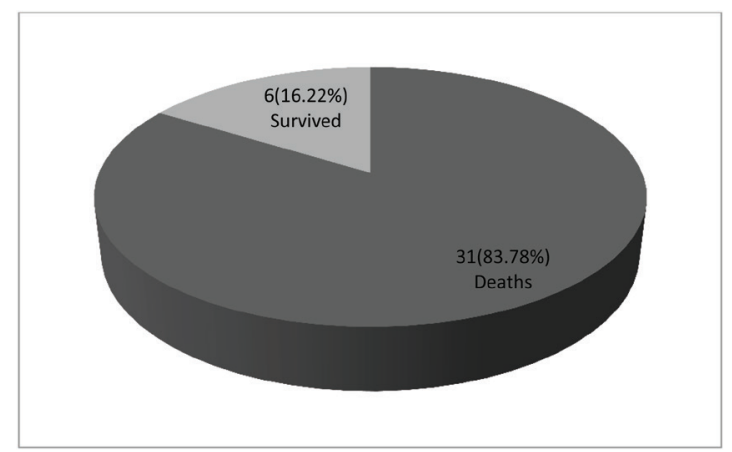

Figure 1 Diagram showing the overall outcome of the 37 patients with traumatic brain injury patients with critical Glasgow coma scale undergoing hemorrhage evacuation craniotomy

predominance with only 9 females. The surgery was performed in emergency operating room below 6 hours after admission to the emergency ward. The most common lesion was EDH, followed by combination of EDH with SDH.

\section{Outcomes}

Surgery was performed in 37 patients. Total 6 of these patients survived and 31 died. 15 patients most commonly underwent surgery for evacuation of an EDH and only 4 of 15 were survived. The other indications for surgery included large contusions with mass effect and midline shift, and decompressive craniectomy for severe brain edema. In 26 cases, a decompressive craniectomy was done concomitantly with the evacuation hemorrhageand10 patients were done cranioplasty autograft.

\section{DISCUSSION}

The term of critical GCS refers to the condition of the patients whose score below or equal to 5 . Critical GCS score is considered highly predictive of death or poor recovery in traumatic brain injury patients. ${ }^{9-11}$ The management of traumatic brain injury especially with critical GCS is complicated and a constant dilemma. Due to the high mortality rate, the management of patients with low score of GCS has been questioned as to whether these patients should be taken surgery as an indication and whether they have any chance to survive after surgery. ${ }^{7,12,13}$

There was limited study to discuss about aggressive treatment especially surgery treatment as management of traumatic brain injury with critical GCS. There is some tendency to treat these patients aggressively because of poor outcome. ${ }^{5,6}$

In our center, we treat these patients aggressively. Compare to a study by Chamoun et al. which also treat blunt head trauma with GCS 3 aggresively, our 
center had very high mortality rate. ${ }^{5}$ The outhors belives that the difference could be due to time to reach the Hospital. Local wisdom may also be influential in our case, where these patient with these conditions conveyed requires a family agreement to refer to our center where it is sometimes very difficult to find a patient family in a short time period.

Although the overall mortality rate of these study was $83.73 \%, 4$ of 15 patients who underwent surgery for evacuation of an EDH survived. High series of patients with EDH and a GCS score of below and equal 5 on presentation are lacking. However, it appears that this subgroup of patients has a significantly higher survival rate than those with other types of intracranial injuries. In general, we believe that patients who present with a GCS score of below and equal 5 due to mass effect from a hematoma (especially an EDH) have a higher chance of survival and functional recovery than patients whose neurological status is mainly caused by SDH and ICH.

Although it is true that the data used are not sufficient to say that a model is robust, this work has shown that it is possible to find patterns in this type of patients, despite the different ranges in the rate of the progress of each one. Furthermore, some data were not available (GCS survive patients when discharge) and several patients were lost to follow-up adding to the limitations of the analysis. Consequently, a simple statistical analysis (rather than a detailed one) was performed to determine the main factors that appeared to influence the outcome.

This study should be validated using another predictive outcome scale of TBI to make a better decision about which is more appropriate to treated aggressively or only for informed counseling of relatives. It requires a larger sample of patients who completed surgery. The present study suggests that recording of patients' data has to be emphasized due to loss of follow up after six months.

\section{CONCLUSION}

In summary, this study summarized that only $16.66 \%$ (6 patients) were survived after surgery. We believe that patients with critical GCS with the score below and equal 5 should be treated aggressively.
Many factors can be main predictive outcome in this patient population. However, the number of patients was too small to draw definite conclusions. Further research is needed to continue this current study, so that the determination for patients with critical GCS can be done in accordance with the provision.

\section{REFERENCES}

1. Joseph, B. et al. Clinical outcomes in traumatic brain injury patients on. 76, 0-3 (2014).

2. Clinical Analysis below ges 8.pdf.

3. Turgeon, A. F. et al. Prognostication in critically ill patients with severe traumatic brain injury: The TBI-Prognosis multicentre feasibility study. BMJ Open 7, 1-8 (2017).

4. Sekhon, M. S. et al. Implementation of Neurocritical Care Is Associated With Improved Outcomes in Traumatic Brain Injury. Can. J. Neurol. Sci. / J. Can. des Sci. Neurol. 44, 350-357 (2017).

5. Roukoz B. Chamoun, M.D., Claudia S. Robertson, M.D., and Shankar P. Gopinath, M.D. Department of Neurosurgery, Baylor College of Medicine, Houston, T. A. Outcome in patients with blunt head trauma and a Glasgow Coma Scale score of 3 at presentationblic Access. J. Neurosurg 111, 683-687 (2009).

6. Rhee, P., Alo, K. \& Chan, L. S. Outcome and Prognostic Factors in Head Injuries With an Admission Glasgow Coma Scale Score of 3. 139, 2002-2004 (2004).

7. Salottolo, K., Stewart Levy, A., Slone, D. S., Mains, C. W. \& Bar-Or, D. The effect of age on glasgow coma scale score in patients with traumatic brain injury. JAMA Surg. 149, 727-734 (2014)

8. Chamoun, R. B., Robertson, C. S. \& Gopinath, S. P. Outcome in patients with blunt head trauma and a Glasgow Coma Scale score of 3 at presentation. J. Neurosurg. 111, 683-7 (2009).

9. Morrison, C. A. et al. An analysis of neurosurgical practice patterns and outcomes for serious to critical traumatic brain injuries in a mature trauma state. J. Trauma Acute Care Surg. 80, 755-763 (2016).

10. Nelson, C. G. Severe Traumatic Brain Injury: A Case Report. 186-191 (2016). doi:10.12659/AJCR.897116

11. Eaton, J., Hanif, A. B., Grudziak, J. \& Charles, A. Epidemiology, Management, and Functional Outcomes of Traumatic Brain Injury in Sub-Saharan Africa. World Neurosurg. 108, 650-655 (2017).

12. Lorena, J. \& Alonzo, M. Predicting the Clinical Outcome in Patients with Traumatic Brain Injury using Clinical Pathway Scores by. (2013).

13. Zuercher, M., Ummenhofer, W., Baltussen, A. \& Walder, B. The use of Glasgow Coma Scale in injury assessment: A critical review. Brain Inj. 23, 371-384 (2009).

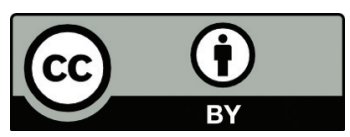

This work is licensed under a Creative Commons Attribution 